\title{
Contribution to fatigue damage prediction of thin shell finite element models
}

\author{
Milan Sága ${ }^{1, *}$, Milan Vaško ${ }^{1}$, Peter Kopas ${ }^{1}$, Wiesława Piekarska $^{2}$, Tomasz Domański ${ }^{2}$, \\ Marcin Kubiak ${ }^{2}$ \\ ${ }^{1}$ University of Žilina, Faculty of Mechanical Engineering, Department of Applied Mechanics, \\ Univerzitná 8215/1, Žilina, Slovakia \\ ${ }^{2}$ Częstochowa University of Technology, Faculty of Mechanical Engineering and Computer Science, \\ Institute of Mechanics and Machine Design Foundations, Dąbrowskiego 73, Częstochowa, Poland
}

\begin{abstract}
The paper deals with the implementation of the chosen fatigue damage criterions into FE analysis. Our study considers a shell finite element structural analysis in conjunction with the multiaxial rainflow counting and the fatigue damage prediction. The computational analysis considers stochastic response under random excitation in time domain and damage calculation based on so called critical plane approach (CPA).
\end{abstract}

Keywords: finite element analysis, multiaxial rainflow counting, fatigue damage

\section{Introduction}

Fatigue damage of structural features is a complex physical process which is governed by a great number of parameters related to, for example, local geometry and material properties of the structural region surrounding the crack growth path. There are different approaches and methods which can be used in fatigue life predictions [1].

It is commonly recognized that it is impossible for a physical model to account for all fatigue influencing parameters, thus a lot of approximate models have been conceived for practical fatigue assessments. In every stadium of fatigue damage cumulation dominates a definite mechanism controlled by more or less known and verified rules. There exists the stage of microplastic process in total volume of material with following stage of fatigue crack nucleation and stage of their growing with more or less detailed zoning. Despite of this research no results have been achieved, which could be considered as successful ones. This applies mainly to the cases of random and combined stress, where today's procedures used in one axis stress analysis fails [2].

There are plenty of hypotheses used for evaluating a degree of damage caused by variable load [3-5]. Life prediction methods which presume homogeneous material (free from cracks, inclusions or defects) at the outset of the investigation can be divided into strain-based (low-cycle fatigue) and stress-based (high-cycle fatigue) methods. This study assumes stress-based approaches use the elastic stress range (or amplitude) as the governing

\footnotetext{
* Corresponding author: milan.saga@,fstroj.uniza.sk

Reviewers: Juraj Gerlici, Marek Macko
} 
load parameter. At a sufficient load level, which may result in a fatigue life of approximately $10^{7}$ cycles, a threshold referred to as the fatigue or endurance limit can be seen for many materials.

Research of mechanism and processes of fatigue failure of materials achieves great advance, however there still doesn't exist general failure model, which should be applicable for different conditions of activity. There is needed an integration for such a procedures into a modern systems of computing aided design (CAD) [6-8] in relationship to methods of strength computing transferred by finite element method (FEM) [9-11].

\section{Thin shell stress calculation}

We will consider well-known shell finite elements (Kirchhoff's or Mindlin's formulation) $[1,12]$. The stiffness parameters depend on material constants and element geometry, mainly on its thickness. At first we have to prepare the stress calculation process. This process is based on the expression of the $j$-th element membrane forces and bending moments (without shear forces) $[13,14]$, i.e.

$$
\left[\begin{array}{lll}
F_{x x} & F_{y y} & F_{x y}
\end{array}\right]_{j}^{T}=\mathbf{F}_{m}^{j}=\int_{S} \mathbf{E}_{m}^{j} \cdot \boldsymbol{\varepsilon}_{m}^{j} d S_{j}=\mathbf{E}_{m}^{j} \cdot \int_{S} \mathbf{B}_{m}^{j} d S_{j} \cdot \mathbf{u}_{L}^{j}=t_{j} \cdot \mathbf{D}_{j} \cdot \mathbf{I}_{m}^{j} \cdot \mathbf{u}_{L}^{j}
$$

and

$$
\left[\begin{array}{lll}
M_{x x} & M_{y y} & M_{x y}
\end{array}\right]_{j}^{T}=\mathbf{M}_{b}^{j}=\int_{S} \mathbf{E}_{b}^{j} \cdot \mathbf{\varepsilon}_{b}^{j} \cdot d S_{j}=\mathbf{E}_{b}^{j} \cdot \int_{S} \mathbf{B}_{b}^{j} \cdot d S_{j} \cdot \mathbf{u}_{L}^{j}=\frac{t_{j}^{3}}{12} \cdot \mathbf{D}_{j} \cdot \mathbf{I}_{b}^{j} \cdot \mathbf{u}_{L}^{j} .
$$

The auxiliary matrices $\mathbf{I}_{m}$ and $\mathbf{I}_{b}$ can be calculated only using the numerical approach. Further details about $\mathbf{E}_{m}, \mathbf{E}_{b}, \mathbf{D}, \mathbf{B}_{m}, \mathbf{B}_{b}, \mathbf{u}_{e l}$ and $t$ are presented in [10,15]. The extreme stress values can be expected at the top or at the bottom surface. Generally, it means

$$
\left[\begin{array}{l}
\left.\left.\boldsymbol{\sigma}_{m b}\right|_{t o p} \boldsymbol{\sigma}_{m b}\right|_{b o t} ^{j}
\end{array}\right]^{j}=\left\{\begin{array}{l}
\sigma_{x x, t o p} \\
\sigma_{y y, t o p} \\
\sigma_{x y, t o p} \\
\sigma_{x x, b o t} \\
\sigma_{y y, b o t} \\
\sigma_{x y, b o t}
\end{array}\right\}^{j}=\left[\begin{array}{cccccc}
1 / t_{j} & 0 & 0 & 6 / t_{j}^{2} & 0 & 0 \\
0 & 1 / t_{j} & 0 & 0 & 6 / t_{j}^{2} & 0 \\
0 & 0 & 1 / t_{j} & 0 & 0 & 6 / t_{j}^{2} \\
1 / t_{j} & 0 & 0 & -6 / t_{j}^{2} & 0 & 0 \\
0 & 1 / t_{j} & 0 & 0 & -6 / t_{j}^{2} & 0 \\
0 & 0 & 1 / t_{j} & 0 & 0 & -6 / t_{j}^{2}
\end{array}\right] \cdot\left\{\begin{array}{l}
F_{x x} \\
F_{y y} \\
F_{x y} \\
M_{x x} \\
M_{y y} \\
M_{x y}
\end{array}\right\}=\left[\begin{array}{l}
\mathbf{A}_{t, t o p} \\
\mathbf{A}_{t, b o t}
\end{array}\right]^{j} \cdot\left\{\begin{array}{l}
\mathbf{F}_{m} \\
\mathbf{M}_{b}
\end{array}\right\}^{j},
$$

or

$$
\boldsymbol{\sigma}_{m b_{-} L}^{j}=\mathbf{C}_{L}^{j} \cdot \mathbf{f}_{L}^{j}
$$

Let's build new material and auxiliary matrices

$$
\mathbf{E}_{m b}=\left[\begin{array}{cc}
t_{j} \cdot \mathbf{I}_{3} & \mathbf{0}_{3} \\
\mathbf{0}_{3} & \frac{t_{j}^{3}}{12} \cdot \mathbf{I}_{3}
\end{array}\right] \cdot\left\{\begin{array}{l}
\mathbf{D} \\
\mathbf{D}
\end{array}\right\}_{j}=\mathbf{D}_{t} \cdot \mathbf{D}_{m b}, \quad \mathbf{I}_{m b}=\left\{\begin{array}{l}
\mathbf{I}_{m}^{j} \\
\mathbf{I}_{b}^{j}
\end{array}\right\},
$$


where the matrix $\mathbf{I}_{3}$ is the classical unit matrix. Then (3) can be rewritten as follows

$$
\begin{aligned}
& \left.\boldsymbol{\sigma}_{j_{-} m b}\right|_{t o p}=\mathbf{A}_{t, t o p} \cdot \mathbf{E}_{m b} \cdot \mathbf{I}_{m b} \cdot \mathbf{u}_{L}^{j}=\mathbf{A}_{t, t o p} \cdot \mathbf{D}_{t} \cdot \mathbf{D}_{m b} \cdot \mathbf{I}_{m b} \cdot \mathbf{u}_{L}^{j}, \\
& \left.\boldsymbol{\sigma}_{j_{-} m b}\right|_{b o t}=\mathbf{A}_{t, b o t} \cdot \mathbf{E}_{m b} \cdot \mathbf{I}_{m b} \cdot \mathbf{u}_{L}^{j}=\mathbf{A}_{t, b o t} \cdot \mathbf{D}_{t} \cdot \mathbf{D}_{m b} \cdot \mathbf{I}_{m b} \cdot \mathbf{u}_{L}^{j} .
\end{aligned}
$$

\section{Chosen approaches for multiaxial fatigue damage calculation}

Let's now focus on the cumulative damage counting by using multiaxial rainflow decomposition of the stress response. It should be noted that the fatigue damage calculation of the machine parts is generally problematic because the results are considerable changed in the principal stresses $[2,4,5]$. Using FE analysis we can get six components of the stress-time function (multiaxial stress) but it is very difficult to obtain an equivalent uniaxial load spectrum by reason of comparison with applied computational fatigue curve.

In our case the rainflow analysis for random stresses known in classic uniaxial form as von Mises or Tresca hypotheses is impossible. It means that the important goal of this part will be to propose some approaches to estimate the high-cycle fatigue damage for multiaxial stresses caused by random vibration analysed structure [10, 16, 17]. Generally we can apply two fundamental approaches for multiaxial rainflow counting:

- Critical Plane Approach (CPA) [13], and

- Integral Approach (IA) [10].

It is well-known that the Wöhler curve (Fig. 1, sometimes called S-2N curve) is basic source of getting information of the material fatigue life. Generally the S-2N curve is statistically evaluating by experimental fatigue curve. This is a graph of the magnitude of a cyclical nominal stress $\sigma_{A}$ against the logarithmic scale of cycles to failure $2 N_{f}$. It is advantage to show it in logarithmical or semi logarithmical coordinates.

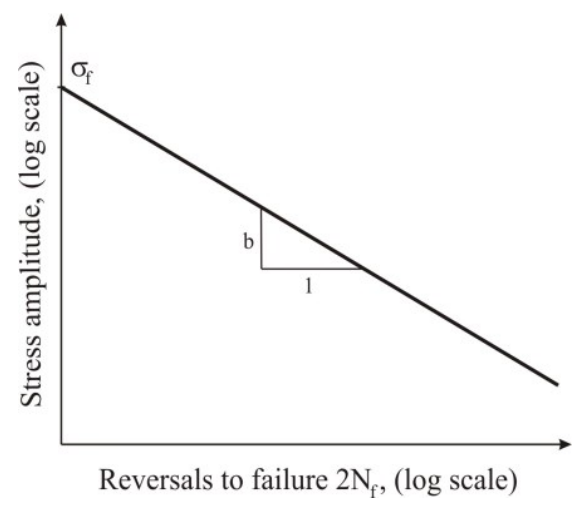

Fig. 1. S-2N curve

The $\sigma_{A}-2 N_{f}$ relation can be written as follows

$$
\sigma_{A}=\sigma_{f} \cdot\left(2 N_{f}\right)^{b}
$$

where $\sigma_{f}$ is the fatigue stress coefficient, $2 N_{f}$ is number of cycles to failure, $b$ is fatigue strength exponent and $\sigma_{A}$ is stress amplitude to failure. 
Some researches the relationship (7) rewrite into following form

$$
\sigma_{A}^{m} \cdot\left(2 N_{f}\right)=K
$$

where $m=-(1 / b)$ and $K=\sigma_{f}^{(-1 / b)}={\sigma_{f}}^{m}$.

Considering mean stress modified version of the stress amplitude (using Goodman, Soderberg, Geber), Eq. (8) can be rewritten as follows

$$
\left\{\sigma_{A} \cdot\left[1-\left(\frac{\sigma_{M}}{R_{F}}\right)^{k}\right]^{-1}\right\}^{m} \cdot\left(2 N_{f}\right)=K .
$$

If $k=1$ and $R_{F}=R_{E}$ (yield stress) the Soderberg's model is used, if $k=1$ and $R_{F}=R_{M}$ (strength limit) the Goodman's model is used and if $k=2$ and $R_{F}=R_{M}$ the Geber's model is used. Using the linear Palmgren-Miner law we can calculate fatigue damage for stress amplitude $\sigma_{A i}$ as follows

$$
d_{i}=\frac{1}{2 N_{f_{-} i}}=\left\{\frac{\sigma_{A i}}{\sigma_{f}} \cdot\left[1-\left(\frac{\sigma_{M}}{R_{F}}\right)^{k}\right]^{-1}\right\}^{m} .
$$

\section{Findley hypothesis}

Findley has assumed the critical plane as a plane with maximum shear stress, i.e. the fatigue equivalent shear stress can be written as follows $[10,18]$

$$
\tau_{A_{-} \text {Fin }}=\tau_{\max }+k \cdot \sigma_{m}
$$

where $k$ is Findley's factor which value for tough metal can be about 0.3 . Using von Mises relationship between normal and shear stresses and classic plane stress analysis for top or bottom element surface it is possible rewrite (9) into following form

$$
\begin{aligned}
& \left.\sigma_{A_{-} F i n}^{j}\right|_{t o p}= \\
& =\sqrt{3} \cdot\left\{\operatorname{sign}\left(\left.\mathbf{T}_{2} \cdot \boldsymbol{\sigma}_{j_{-} m b}\right|_{t o p}\right) \cdot\left[\left(\left.\boldsymbol{\sigma}_{j_{-} m b}\right|_{t o p}\right)^{T} \cdot \mathbf{T}_{1} \cdot\left(\left.\boldsymbol{\sigma}_{j_{-} m b}\right|_{t o p}\right)\right]^{-1 / 2}+\left.k \cdot \mathbf{T}_{2} \cdot \boldsymbol{\sigma}_{j_{-} m b}\right|_{t o p}\right\}, \\
& \left.\sigma_{A_{-} F i n}^{j}\right|_{b o t}= \\
& =\sqrt{3} \cdot\left\{\operatorname{sign}\left(\left.\mathbf{T}_{2} \cdot \boldsymbol{\sigma}_{j_{-} m b}\right|_{b o t}\right) \cdot\left[\left(\left.\boldsymbol{\sigma}_{j_{-} m b}\right|_{b o t}\right)^{T} \cdot \mathbf{T}_{1} \cdot\left(\left.\boldsymbol{\sigma}_{j_{-} m b}\right|_{b o t}\right)\right]^{-1 / 2}+\left.k \cdot \mathbf{T}_{2} \cdot \boldsymbol{\sigma}_{j_{-} m b}\right|_{b o t}\right\} .
\end{aligned}
$$

where

$$
\mathbf{T}_{1}=\left[\begin{array}{ccc}
0.25 & -0.25 & 0 \\
-0.25 & 0.25 & 0 \\
0 & 0 & 1
\end{array}\right] \quad \text { and } \quad \mathbf{T}_{2}=\left[\begin{array}{lll}
0.5 & 0.5 & 0
\end{array}\right]
$$


The damage calculation can be realised using Eq. (10). Presented relationships have been applicable for FE analyses. Numerical and experimental tests have confirmed that the factor $k=0.3$ was overstated $[4,13]$ by author.

\section{Dang Van hypothesis}

Dang Van again has assumed the critical plane with shear stress but with difference in factor $k$, which can be calculated from normal and shear fatigue limit, i.e.

$$
\tau_{A_{-} D V}=\tau_{\max }+k \cdot p_{m}=\frac{\sigma_{1}-\sigma_{3}}{2}+\frac{\tau_{C}-\frac{\sigma_{C}}{2}}{\frac{\sigma_{C}}{\sqrt{3}}} \cdot \frac{\sigma_{1}+\sigma_{2}+\sigma_{3}}{3},
$$

where $\tau_{C}$ is shear (torsional) fatigue limit, $\sigma_{C}$ is normal (axial) fatigue limit, $\sigma_{1}, \sigma_{2}, \sigma_{3}$ are principal stresses. Relationship (11) is possible to use like that

$$
\sigma_{A_{-} D V}=\sqrt{3} \cdot\left[\operatorname{sign}\left(\frac{\sigma_{1}+\sigma_{3}}{2}\right) \cdot \frac{\sigma_{1}-\sigma_{3}}{2}+\frac{\tau_{C}-\frac{\sigma_{C}}{2}}{\frac{\sigma_{C}}{\sqrt{3}}} \cdot \frac{\sigma_{1}+\sigma_{2}+\sigma_{3}}{3}\right] .
$$

Using von Mises hypothesis we can get [4]

$$
\frac{\tau_{C}-\frac{\sigma_{C}}{2}}{\frac{\sigma_{C}}{\sqrt{3}}} \approx \frac{\tau_{C}-\frac{\sqrt{3} \cdot \tau_{C}}{2}}{\frac{\sqrt{3} \cdot \tau_{C}}{3}} \approx 0,232 .
$$

Using by application of the shell stress theory and Eqs. 6a,b we can obtain

$$
\begin{aligned}
& \left.\sigma_{A_{-} D V}^{j}\right|_{\text {top }}= \\
= & \sqrt{3} \cdot\left\{\operatorname{sign}\left(\left.\mathbf{T}_{3} \cdot \boldsymbol{\sigma}_{j_{-} m b}\right|_{\text {top }}\right)\left[\left(\left.\boldsymbol{\sigma}_{j_{-} m b}\right|_{t o p}\right)^{T} \cdot \mathbf{T}_{1} \cdot\left(\boldsymbol{\sigma}_{j_{-} m b} \mid t_{t o p}\right)\right]^{-1 / 2}+0,\left.232 \cdot \mathbf{T}_{3} \cdot \boldsymbol{\sigma}_{j_{-} m b}\right|_{t o p}\right\}, \\
& \left.\sigma_{A_{-} D V}^{j}\right|_{b o t}= \\
= & \sqrt{3} \cdot\left\{\operatorname{sign}\left(\left.\mathbf{T}_{3} \cdot \boldsymbol{\sigma}_{j_{-} m b}\right|_{b o t}\right) \cdot\left[\left(\left.\boldsymbol{\sigma}_{j_{-} m b}\right|_{b o t}\right)^{T} \cdot \mathbf{T}_{1} \cdot\left(\left.\boldsymbol{\sigma}_{j_{-} m b}\right|_{b o t}\right)\right]^{-1 / 2}+0,\left.232 \cdot \mathbf{T}_{3} \cdot \boldsymbol{\sigma}_{j_{-} m b}\right|_{b o t}\right\} .
\end{aligned}
$$

Relations (12) and (17) present equivalent stresses applicable for rainflow decomposition for both proportional and non-proportional loading. The cumulative damage calculation can be realised using Eq. (10) again.

\section{HMH modified hypothesis}

Applying von Mises equivalent stress for CPA we can obtain following relationship

$$
\sigma_{A_{-} H M H}=\operatorname{sign}\left[\sigma\left(\mathbf{n}_{C P A}\right)\right] \cdot \sqrt{\sigma\left(\mathbf{n}_{C P A}\right)^{2}+3 \cdot \tau\left(\mathbf{n}_{C P A}\right)^{2}},
$$


or in detail

$$
\begin{aligned}
& \left.\sigma_{A_{-} H M H}^{j}\right|_{t o p}\left(\mathbf{n}_{C P A}\right)= \\
& =\operatorname{sign}\left[\left.\left.\boldsymbol{\sigma}_{j_{-} m b}^{T}\right|_{t o p}\left(\mathbf{n}_{C P A}\right) \cdot \mathbf{T}_{\sigma} \cdot \boldsymbol{\sigma}_{j_{-} m b}\right|_{t o p}\left(\mathbf{n}_{C P A}\right)\right] \cdot\left[\left.\left.\boldsymbol{\sigma}_{j_{-} m b}^{T}\right|_{t o p}\left(\mathbf{n}_{C P A}\right) \cdot \mathbf{T}_{4} \cdot \boldsymbol{\sigma}_{j_{-} m b}\right|_{t o p}\left(\mathbf{n}_{C P A}\right)\right]^{-1 / 2}, \\
& \left.\sigma_{A_{-} H M H}^{j}\right|_{b o t}\left(\mathbf{n}_{C P A}\right)= \\
& =\operatorname{sign}\left[\left.\left.\boldsymbol{\sigma}_{j_{-} m b}^{T}\right|_{b o t}\left(\mathbf{n}_{C P A}\right) \cdot \mathbf{T}_{\sigma} \cdot \boldsymbol{\sigma}_{j_{-} m b}\right|_{b o t}\left(\mathbf{n}_{C P A}\right)\right] \cdot\left[\left.\left.\boldsymbol{\sigma}_{j_{-} m b}^{T}\right|_{b o t}\left(\mathbf{n}_{C P A}\right) \cdot \mathbf{T}_{4} \cdot \boldsymbol{\sigma}_{j_{-} m b}\right|_{b o t}\left(\mathbf{n}_{C P A}\right)\right]^{-1 / 2},
\end{aligned}
$$

where

$$
\mathbf{T}_{4}=\left[\begin{array}{ccc}
1 & -0.5 & 0 \\
-0.5 & 1 & 0 \\
0 & 0 & 3
\end{array}\right] \quad \text { and } \quad \mathbf{T}_{\sigma}=\left[\begin{array}{ccc}
\sigma_{x} & \tau_{x y} & 0 \\
\tau_{x y} & \sigma_{y} & 0 \\
0 & 0 & 0
\end{array}\right]
$$

In this case it should be noted that computational approach depends on a searching process of a critical plane normal vector $\mathbf{n}_{C P A}$. By reason rainflow analysis it is very important to know the sign of the calculated equivalent stress therefore the sign of this stress will be defined by sign of normal component. For searching process was used optimizing tools in Matlab [7] and optimizing problem for cumulative damage function is usually formulated as follows

$$
\begin{aligned}
& \left.D_{i_{-} \max }\right|_{H M H}=\max \left[\sum_{j=1}^{m c} d_{j}\left(\mathbf{n}_{C P A}\right)\right]= \\
& =\max \left\{\left\{\frac{\left.\sigma_{A_{-} H M H}\right|_{b o t}\left(\mathbf{n}_{C P A}\right)_{j}}{\sigma_{f}} \cdot\left[1-\left(\frac{\left.\sigma_{M_{-} H M H}^{i}\right|_{b o t}\left(\mathbf{n}_{C P A}\right)_{j}}{R_{F}}\right)^{k}\right]^{-1}\right\}\right.
\end{aligned}
$$

for unknown vector $\mathbf{n}_{C P A}$ and stresses on bottom surface. The same computational process we can have to realise on top element surface.

\section{Damage calculation using $I A$}

The fundamental idea is to count rainflow cycles on all linear combinations of the stress random vector components [10], i.e.

$$
\begin{aligned}
& \sigma_{A_{-} M R F}(t)= \\
& =c_{1} \cdot \sigma_{x}(t)+c_{2} \cdot \sigma_{y}(t)+c_{3} \cdot \sigma_{z}(t)+c_{4} \cdot \tau_{x y}(t)+c_{5} \cdot \tau_{y z}(t)+c_{6} \cdot \tau_{z x}(t)
\end{aligned}
$$

on the assumption that the parameters $c_{i}$ belong to a hypersphere $\sum_{i=1}^{n} c_{i}^{2}=1$. Practically if the stress state is biaxial (e.g. thin shell finite element) the stress components can be written under the form of three dimension vector $\sigma=\left[\sigma_{x}, \sigma_{y}, \tau_{x y}\right]^{\mathrm{T}}$ and the equivalent stress will be calculated as follows

$$
\sigma_{A_{-} M R F}(t)=c_{1} \cdot \sigma_{x}(t)+c_{2} \cdot \sigma_{y}(t)+c_{3} \cdot \tau_{x y}(t)=\mathbf{c} \cdot \boldsymbol{\sigma}
$$


on condition that $c_{1}^{2}+c_{2}^{2}+c_{3}^{2}=1$. In the case of shell element we can obtain again following relationships

$$
\left.\sigma_{A_{-} M R F}^{j}\right|_{t o p}(\mathbf{c})=\left.\mathbf{c} \cdot \boldsymbol{\sigma}_{j_{-} m b}\right|_{t o p}(\mathbf{c}) \text { and }\left.\sigma_{A_{-} M R F}^{j}\right|_{b o t}(\mathbf{c})=\left.\mathbf{c} \cdot \boldsymbol{\sigma}_{j_{-} m b}\right|_{b o t}(\mathbf{c}) .
$$

Hence the next goal will be to find extreme value of the estimated damage for vector $\mathbf{c}$ and $i$-th element, i.e.

$$
\left.D_{i_{-} \max }\right|_{M R F}=\max \left[\sum_{j=1}^{m c} d_{j}(\mathbf{c})\right]=\max \left\{\left\{\frac{\left.\sigma_{A_{-} M R F}^{i}\right|_{b o t}(\mathbf{c})_{j}}{\sigma_{f}} \cdot\left[1-\left(\frac{\left.\sigma_{M_{-} M R F}^{i}\right|_{b o t}(\mathbf{c})_{j}}{R_{F}}\right)^{k}\right]^{-1}\right\},\right.
$$

where $\left.D_{i-\max }\right|_{M R F}$ is the maximum value of the cumulative damage for $i$-th element, $2 N_{i}$ is the number of cycles to failure, $m c$ is the number of cycles after rain-flow decomposition of the stress. Naturally we have to observe the normality condition for c using following transformation

$$
\mathbf{c}=\frac{\mathbf{c}^{\prime}}{\sqrt{\mathbf{c}^{\prime T} \cdot \mathbf{c}^{\prime}}} .
$$

\section{Testing example}

Let's solve fatigue damage of the model of ribbed beam as a shell construction created in FEA software ADINA (Fig. 3). The real geometry of this component is shown in Fig. 2. On this model there was analyzed rate of cumulative fatigue damage with help of presented CPA methods. We considered calculation with the chosen multiaxial criterions which were applied for 16 identical random loading processes (2000 random values - Figs. 4 and 5): Dang Van criterion; Findley criterion and Von Mises criterion.

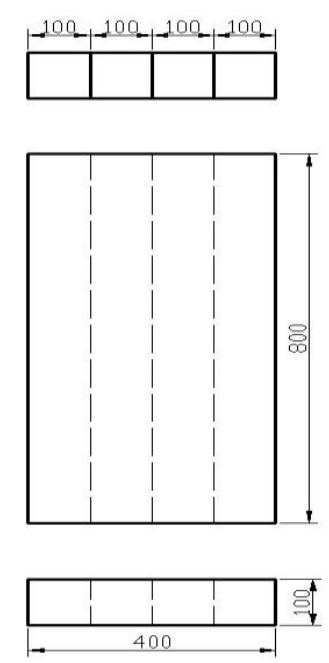

Fig. 2. Proportions of ribbed beam with wall thickness of $6 \mathrm{~mm}$ 


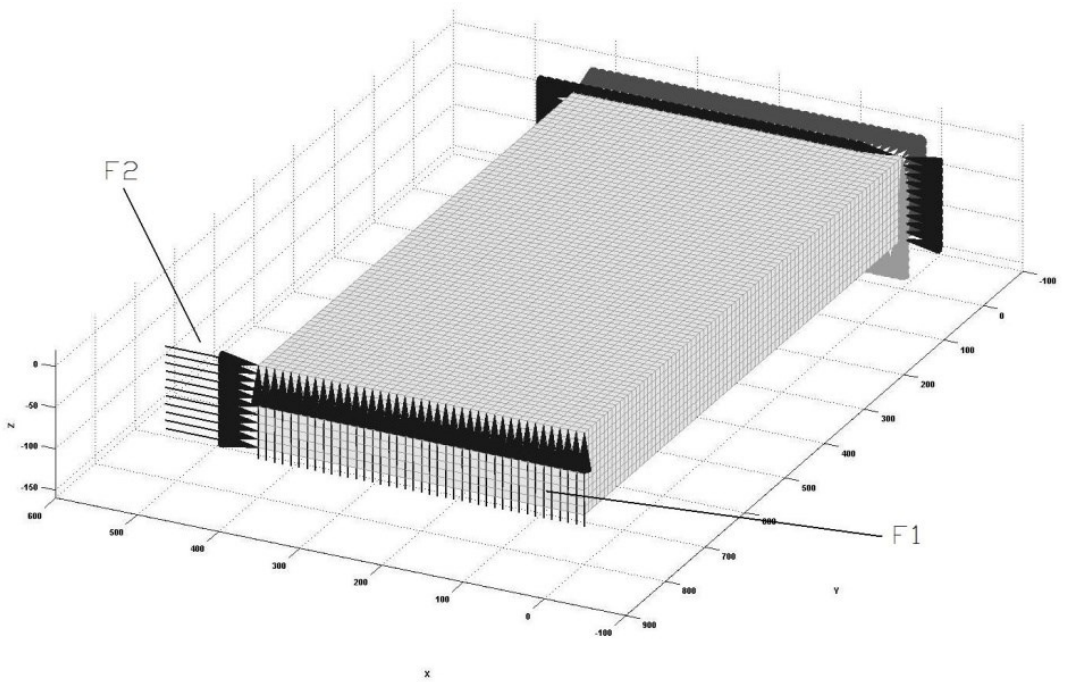

Fig. 3. Three-Dimensional model of ribbed beam

The Fig. 3 represents 3D model of ribbed beam and its mesh with detail of loading. We used the following parameters in finite element model: used material was typical construction steel with Young's modulus $E=2.1 \cdot 10^{11} \mathrm{~Pa}$ and Poisson number $\mu=0.3$. During the FE meshing it was used thin shell finite elements with four nodes. The model was excited under two stochastic forces with Gauss distribution. Stochastic character of this forces we can describe as follows: mean $(F 1)=12000 \mathrm{~N}$, standard deviation $(F 1)=8000 \mathrm{~N}$ at direction of $z$ axis and mean $(F 2)=5000 \mathrm{~N}$, standard deviation $(F 2)=10000 \mathrm{~N}$ at direction of $x$ axis. Time functionality of the forces $F 1$ and $F 2$ are shown in Figs. 4 and 5.

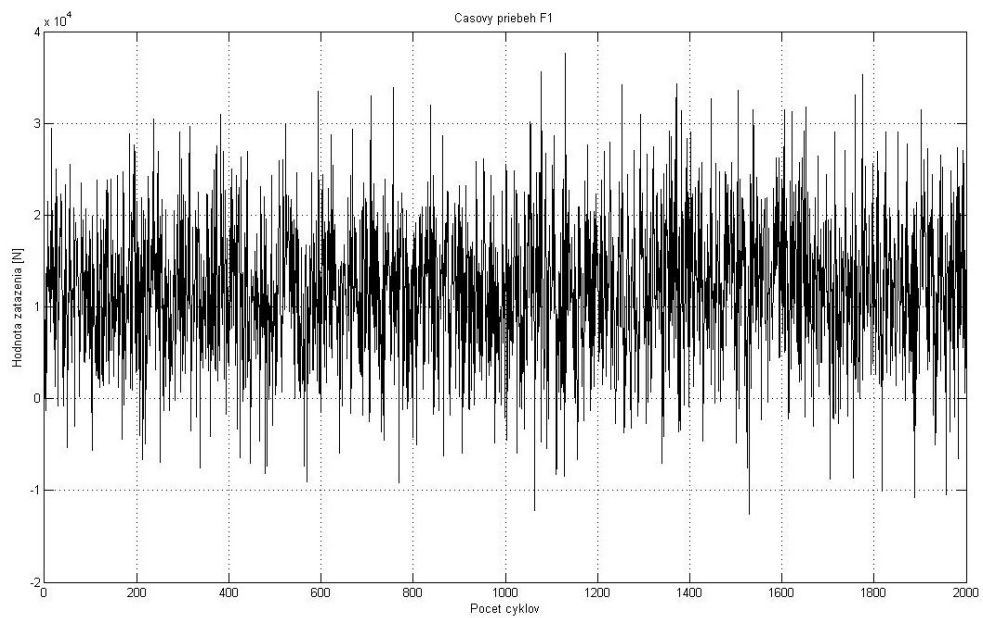

Fig. 4. Time course function of loading force $F 1$ 


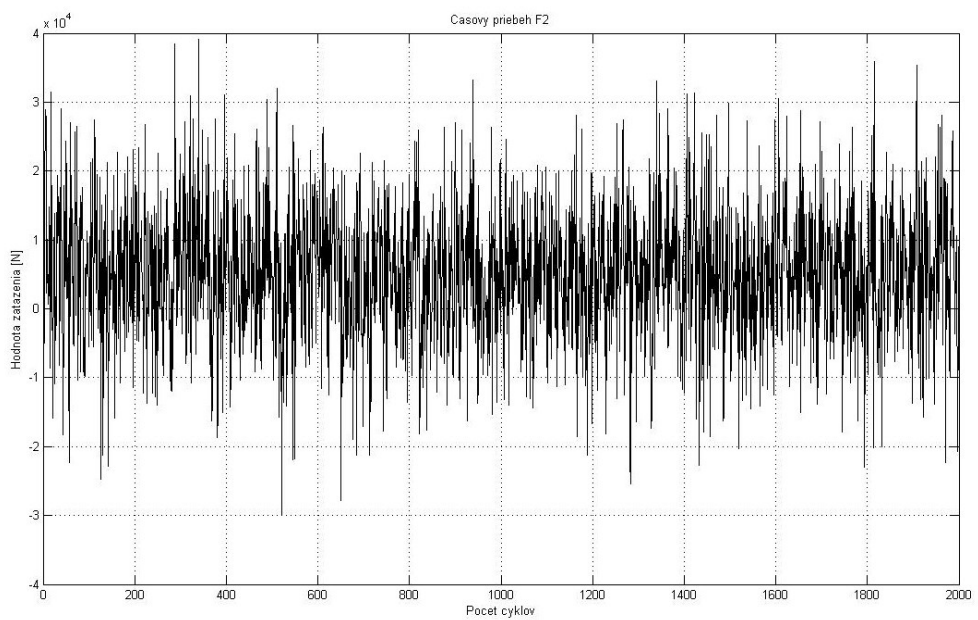

Fig. 5. Time course function of loading force $F 2$

\section{Cumulative Damage Calculations}

In Table 1 is shown estimate of cumulative fatigue damage for ribbed beam. Graphical representations of cumulative fatigue damage of analyzed shell structure for three chosen hypothesis are shown in Fig. 6, Fig. 7 and Fig. 8.

Table 1. Cumulative damage for different multiaxial criterions

\begin{tabular}{|c|c|c|}
\hline Used method & $\begin{array}{c}\text { Node with maximal damage value } \\
\text { [number] }\end{array}$ & $\begin{array}{c}\text { Cumulative fatigue damage } \\
\text { [cycles] }\end{array}$ \\
\hline Dang Van & 1 & $5.2883 \cdot 10^{-4}$ \\
\hline Findley & 1 & $5.303 \cdot 10^{-4}$ \\
\hline Von Mises & 1 & $1.665 \cdot 10^{-2}$ \\
\hline
\end{tabular}

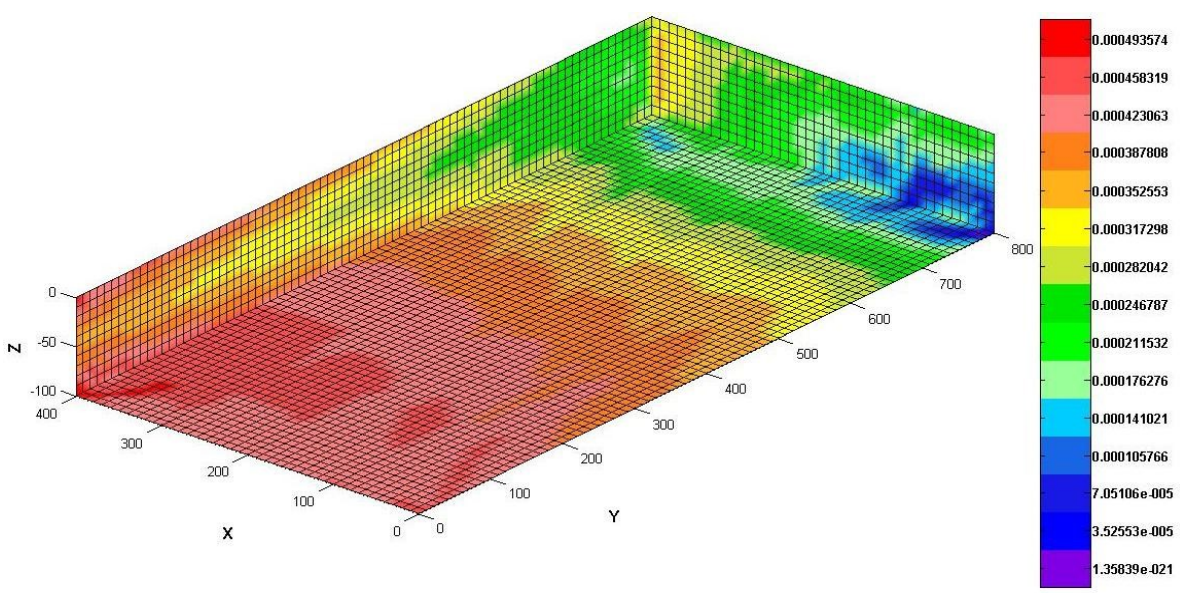

Fig. 6. Distribution of the cumulative fatigue damage using Dang Van criterion 

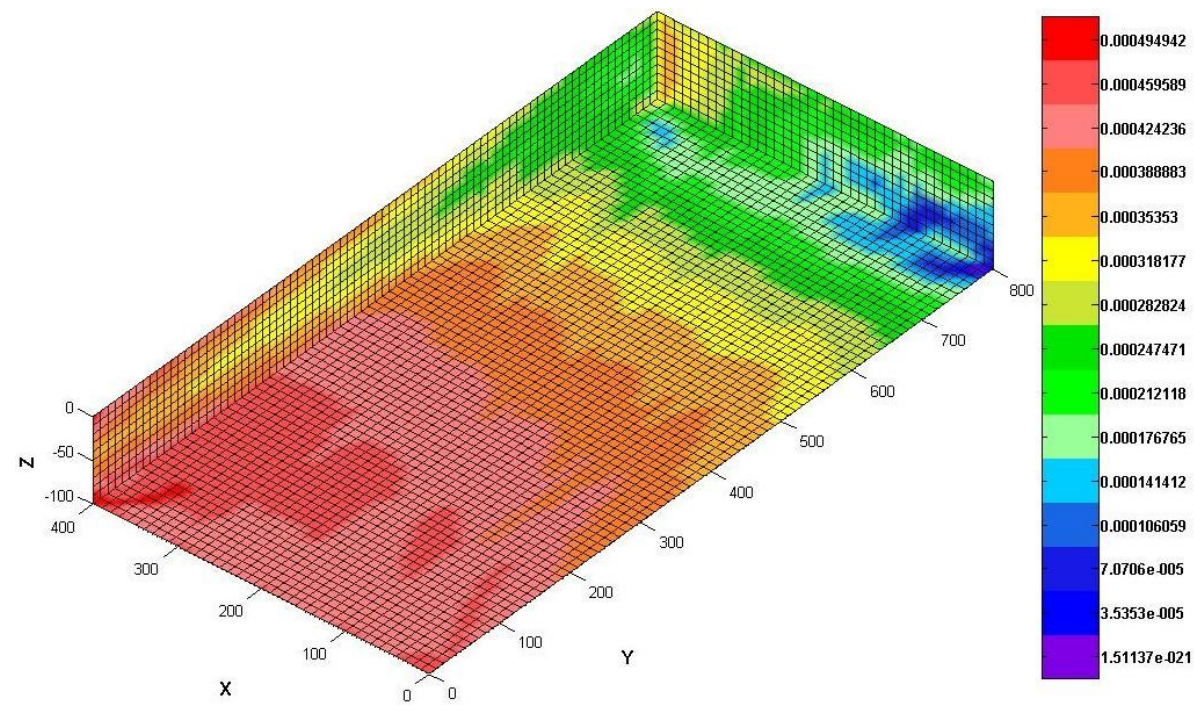

Fig. 7. Distribution of the cumulative fatigue damage using Findley criterion
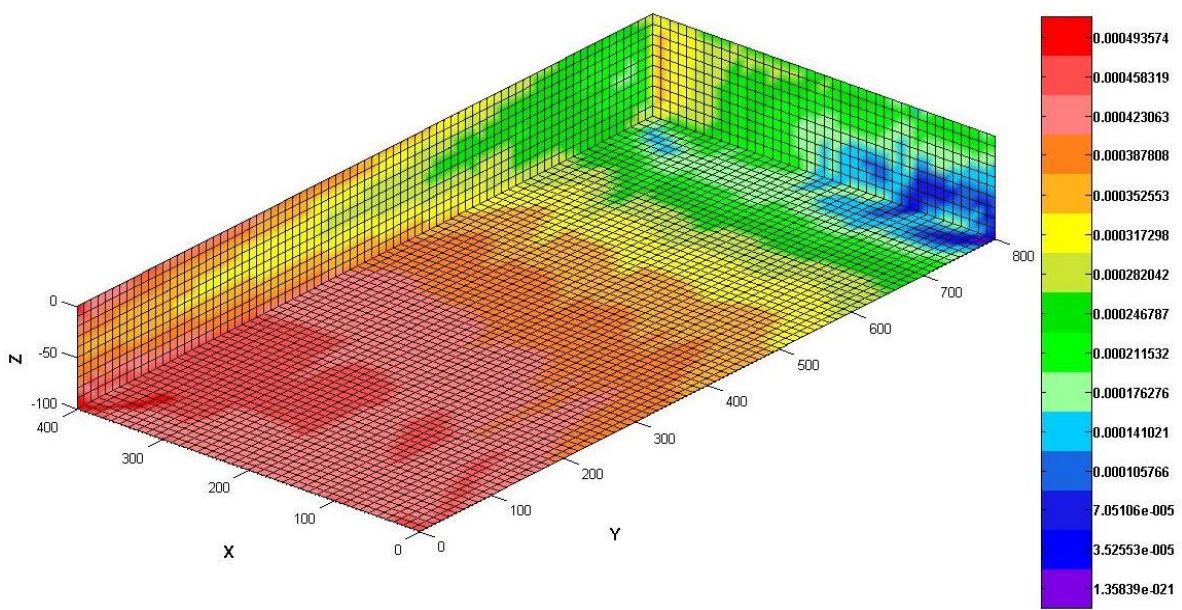

Fig. 8. Distribution of the cumulative fatigue damage using von Mises criterion

Our computational analysis confirms fact that the results of cumulative fatigue damage of ribbed beam is for Dang Van and Findley criterion very similar. The most difference was observed in von Mises criterion which also gave the worst results. Average value of obtained computational results was 0.0059 and standard deviation has value 0.0093 .

\section{Conclusion}

The goal of this study has been to realized an overview and propose of possibilities of computational fatigue damage (life) prediction of modeled shell structure with help of finite element method. There was used a software facility interface between MATLAB and ADINA software. 
They were also presented and compared different methodology about prediction of multiaxial fatigue criterions. Our benefit has been in application of the algorithmization process of the fatigue failure computational prediction in shell finite element models.

This work has been supported by the Slovak Research and Development Agency under the contract No. APVV-14-0096.

\section{References}

1. M. Balda, J. Svoboda, V. Fröhlich, Using hypotheses for calculating fatigue lives of parts exposed to combined random loads. Engineering Mechanics 10 (5), 12-15 (2003)

2. C. Chu, Multiaxial fatigue life prediction methods in the ground vehicle industry. Int. J. Fatigue 19 (1), 325-330 (1997)

3. D. F. Socie, Multiaxial fatigue damage models. J. Eng. Mater. Technol. 1069, 293-298 (1987)

4. X. Pitoiset, A. Preumont, A. Kernilis, Tools for a Multiaxial Fatigue Analysis of Structures Submitted to Random Vibrations. Proceedings European Conference on Spacecraft Structures Materials and Mechanical Testing, Germany, 1-6 (1998)

5. A. Carpinteri, A. Spagnoli, S. Vantadori, A multiaxial fatigue criterion for random loading. Fatigue and Fracture of Engineering Materials and Structures 26 (6 - Special Issue), 515-522 (2003)

6. J. Flizikowski, M. Macko, J. Czerniak, A. Mroziński, Implementation of genetic algorithms into development of mechatronic multi-edge's grinder design. ASME 2011, Int. Mech. Eng. Congress and Exposition, IMECE, (2011)

7. M. Macko, J. Flizikowski, Z. Szczepański, K. Tyszczuk, G. Śmigielski, A. Mroziński, J. Czerniak, A. Tomporowski, CAD/CAE applications in mill's design and investigation. Proc. of the $13^{\text {th }}$ Int. Scientific Conf. on Computer Aided Engineering: Lecture Notes in Mech. Eng., Chapt. 35, Springer Intern. Publ. AG, 343-351 (2017)

8. G. Domek, A. Kołodziej, Design of the tendon structure in timing belts. Procedia Engineering 136, 365-369 (2016)

9. M. Sága, Š. Medvecký, M. Kopecký, The effective algorithm for discrete structural mass minimising subjected to fatigue life. $6^{\text {th }}$ World Congress on Structural and Multidisciplinary Optimization, Rio de Janeiro, Brasil, (full paper on CD)

10. M. Sága, M. Vaško, P. Pecháč, Chosen numerical algorithms for interval finite element analysis. Procedia Engineering 96, 400-409 (2014)

11. J. Gerlici, T. Lack, Modified HHT method for vehicle vibration analysis in time domain utilisation. Applied mechanics and materials 486, 396-405 (2014)

12. T. Lack, J. Gerlici, Rail vehicles brake components test bench utilisation. Applied mechanics and materials 486, 379-386 (2014)

13. M. Dudziak, G. Domek, A. Kołodziej, K. Talaśka, Contact Problems Between the Hub and the Shaft with a Four-Angular Shape of Cross-Section for Different Angular Positions. Applied Mechanics and Materials 816, 54-62 (2015)

14. R. Halama, J. Sedlák, M. Fusek, Z. Poruba, Uniaxial and biaxial ratcheting of ST52 steel under variable amplitude loading-experiments and modeling. Procedia Engineering 101 (C), 185-193 (2015) 
15. V. Dekýš, A. Sapietová, O. Števka, Understanding of the dynamical properties of machines based on the interpretation of spectral measurements and FRF. Experimental stress analysis, Applied Mechanics and Materials 486, 106-112 (2014)

16. J. Vavro, J. Vavro Jr., P. Kováčiková, R. Bezdedová, The optimisation of the materials properties for the passenger cars in dependence on defect distribution at the dynamic loading. Procedia Engineering 136, 114-119 (2016)

17. J. Vavro, J. Vavro Jr., P. Kováčiková, P. Kopas, M. Handrik, Simulation and analysis of defect distribution in passenger car tire under dynamic loading. Applied Mechanics and Materials 611, 544-547 (2014)

18. R. Halama, M. Fusek, Z. Poruba, Influence of mean stress and stress amplitude on uniaxial and biaxial ratcheting of ST52 steel and its prediction by the Abdel KarimOhno model. International Journal of Fatigue 91, 313-321 (2016) 